\title{
Local anesthetic systemic toxicity: proposed mechanisms for lipid resuscitation and methods of prevention
}

\author{
Yutaka Oda ${ }^{1}$ ] \\ Received: 10 April 2019 / Accepted: 24 April 2019 / Published online: 3 May 2019 \\ (c) Japanese Society of Anesthesiologists 2019
}

Keywords Insulin signaling $\cdot$ Lipid $\cdot$ Local anesthetics $\cdot$ Systemic toxicity $\cdot$ Ultrasound

\section{Introduction}

"Practical guide for the management of systemic toxicity caused by local anesthetics" has been published by the Japanese Society of Anesthesiologists [1]. This guide addresses anesthetic management performed by members of our society and can also be referenced during any procedure performed in Japan including the use of local anesthetics. I believe that this guide would contribute to the improvement and progress of anesthesia practice. Although local anesthetic systemic toxicity is a fatal complication, its detailed mechanisms have not been fully elucidated. Recently, several new findings have been reported concerning its toxicity and resuscitation by lipid emulsion. Hereby, I briefly summarize the investigations currently in progress and refer to the methods developed to prevent local anesthetic systemic toxicity.

\section{Mechanisms of the central nervous system toxicity of local anesthetics}

From the report by Tanaka and Yamasaki [2], the mechanisms by which local anesthetics induce central nervous system toxicity have been attributed to the blockade of inhibitory neurons at lower concentration than those blocking excitatory neurons. However, its detailed mechanisms remain unclear. Notably, the threshold plasma concentration of local anesthetics for inducing central nervous system toxicity is lower than that for triggering cardiovascular toxicity. Previous studies have shown that local anesthetics inhibit endogenous glutamate release at lower concentrations than

Yutaka Oda

yutakaodayutaka@gmail.com

1 Department of Anesthesiology, Osaka City Juso Hospital, 2-12-27, Nonaka-kita, Yodogawa-ku, Osaka 532-0034, Japan those blocking voltage-gated sodium channel [3], suggesting that glutamate modulates neuronal excitotoxicity.

Recently, Xing et al. [4] performed a series of experiments using individually cultured rat hippocampal neurons, astrocytes and cocultured astrocytes/neurons. They found that (1) both bupivacaine and ropivacaine had different effects on glutamate-induced increase in intracellular calcium concentration between astrocytes and neurons; (2) bupivacaine produced a decrease in the mitochondrial membrane potential by opening the mitochondrial permeability transition pore only in monocultured astrocytes or astrocytes cocultured with neurons, but not in neurons; (3) bupivacaine dose dependently increased reactive oxygen species in astrocytes, not in neurons. These results may suggest that bupivacaine selectively impairs astrocyte mitochondrial function and thereby suppresses glutamate uptake, which indirectly potentiates glutamate-induced excitation in neurons. Although the direct clinical relevance of these results to the manifestation of toxic symptoms is not clear, it is very unique to focus on astrocyte as well as neurons for investigating the mechanism of the central nervous system toxicity.

\section{Recent advances in the mechanism for lipid resuscitation from cardiovascular toxicity of local anesthetics}

The effectiveness of intravenous lipid emulsion for resuscitation from local anesthetic systemic toxicity has been established by numerous reports [5]. It provides a multimodal resuscitation benefit that includes both scavenging and nonscavenging components [6]. 


\section{Scavenging effect}

Scavenging effect has been known as "lipid sink", which was initially presented as a possible mechanism, based on an in vitro study by Weinberg et al. [7]. Lipid sink has been regarded as a representative mechanism of lipid resuscitation, because lipid is useful as an antidote to non-anesthetic lipophilic agents including cardiovascular as well as psychiatric agents [5]. However, no pharmacokinetic study to validate the "lipid sink" theory has been performed before 2010 . The difficulty in quantitative measurement of local anesthetics, particularly proteinunbound fraction in lipid-containing blood, would be one of the reasons [8]. Subsequently, the question "whether resuscitation from cardiac arrest within such a short period (approximately $<4 \mathrm{~min}$ ) is able to be explained by merely a decrease of local anesthetics concentration" still remained.

From 2010, several pharmacokinetic studies provided critical information. Shi et al. [9] examined the pharmacokinetics of bupivacaine with/without lipid emulsion in rats under general anesthesia with sevoflurane. They found that elimination half-life of plasma total bupivacaine was shortened, and systemic clearance was increased by lipid emulsion; concentrations of bupivacaine was approximately $30 \%$ lower in the heart and brain and 50\% higher in the liver in rats receiving lipid than controls. These results demonstrate that lipid extracted bupivacaine from the brain and heart, then transported it to the liver for elimination, suggesting that lipid exerts not only as a "sink" but as a "scavenger". However it was still not possible to explain such rapid and almost complete recovery of cardiac function by lipid emulsion, implying the involvement of nonscavenging effect.

\section{Nonscavenging effect}

Numerous studies have implicated the involvement of nonscavenging effect of lipid emulsion for local anesthetic systemic toxicity from the initial stage of research. Despite continuing interest in nonscavenging effects, scavenging effects paradoxically proved an important confounder and delayed experimental confirmation of such effects. Several reports published after 2010 strongly suggest a significant role of nonscavenging effect in lipid resuscitation. Gosmanov et al. [10] showed that lipid emulsion drives vasoconstriction and increases blood pressure without contemporaneous drug toxicity, possibly by inhibiting endothelial cell function in healthy subjects. Partownavid et al. [11] demonstrated that free fatty acid oxidation is essential for successful recovery of bupivacaine-induced cardiotoxicity by lipid emulsion in vivo and in mitochondrial suspension.
Notably, a new step was made by Kuo et al. [12] using a physiologically based pharmacokinetic model. They demonstrated that scavenging effect alone was likely not sufficient to explain the prompt and robust recovery of cardiovascular parameters. Fettiplace et al. [13] improved this model and has shown that nonscavenging effects consisting of both a volume and a cardiotonic effect are responsible for resuscitation. They have also shown that recovery of aortic flow and blood pressure occurs approximately within 4 min after starting infusion of lipid emulsion in vivo, and suggested that a rapid cell signaling process might be involved in this procedure [14].

A series of molecular-based studies has shown that bupivacaine induces cell death at subclinical concentrations by suppressing activation of protein kinase B (Akt) [15], a critical node within insulin-related kinase signaling and involved in the regulation of intracellular $\mathrm{Ca}^{++}$handling as well as myocardial contractility [16]. Fettiplace et al. [17] proved that acute bupivacaine cardiotoxicity affects insulin signaling by dephosphorylation of Akt and by phosphorylation of 5 '-adenosine monophosphate, another controller of glucose homeostasis. This process in turn activates Akt signaling in accordance with a decrease of cardiac bupivacaine content, leading to an increase of myocardial contractility. Of importance, lipid emulsion itself enhances the phosphorylation of Akt and cotreatment with bupivacaine sequentially activates Akt signaling, suggesting the involvement of insulin signaling in both bupivacaine-induced cardiotoxicity and antidote effect of lipid emulsion.

\section{How to reduce the incidence of local anesthetic systemic toxicity}

For preventing systemic toxicity from local anesthetics, a small-dose, divided administration, use of local anesthetics with less toxicity, performing an aspiration test and using ultrasound imaging to assess the location of the needle or catheter have been suggested [1]. Besides using less-toxic anesthetics such as ropivacaine and levobupivacaine, various techniques, e.g., liposomal bupivacaine and transdermal lidocaine delivery system, have been performed to prolong the effect of local anesthetics and to minimize the required dose [18]. Low molecular weight dextran is also effective in reducing systemic absorption of local anesthetics and in reducing plasma concentration [19]. Liposomal bupivacaine is commercially available in the USA for local infiltration anesthesia and brachial plexus block. Although liposomal bupivacaine is characterized by a long duration of action, careful observation is essential for detecting systemic toxicity occurring long after administration, particularly when used in combination with other local anesthetics to facilitate the onset of analgesic effect [20]. 
Recently, ultrasound-guided technique for regional anesthesia has been widespread and is useful for increasing the success rate and for reducing the risk of intravascular injection compared with the conventional landmark method [21]. During ultrasound-guided nerve block, precise placement of the needle to the target site is important; however, accurate estimation of the spread of local anesthetic has been difficult because its solution is hypoechoic. Sasaki et al. [22] developed a method for applying perfluorobutane, a contrast medium conventionally used for diagnosing hepatic and breast cancers, to ultrasound-guided nerve block. The authors demonstrated an excellent agreement of the spread of perfluorobutane measured on the ultrasound image with direct visual inspection in a cadaver study. They also confirmed no adverse effect of co-administered lidocaine and perfluorobutane in an animal study. The editor would expect such a safe method will prevail to decrease the incidence of local anesthetic-induced systemic toxicity.

\section{References}

1. Safety Committee of Japanese Society of Anesthesiologists. Practical guide for the management of systemic toxicity caused by local anesthetics. J Anesth. 2019;33:1-8.

2. Tanaka K, Yamasaki M. Blocking of cortical inhibitory synapses by intravenous lidocaine. Nature. 1966;209:207-8.

3. Lin TY, Chung CY, Lu CW, Huang SK, Shieh JS, Wang SJ. Local anesthetics inhibit glutamate release from rat cerebral cortex synaptosomes. Synapse. 2013;67:568-79.

4. Xing Y, Zhang N, Zhang W, Ren LM. Bupivacaine indirectly potentiates glutamate-induced intracellular calcium signaling in rat hippocampal neurons by impairing mitochondrial function in cocultured astrocytes. Anesthesiology. 2018;128:539-54.

5. Cave G, Harvey M, Willers J, Uncles D, Meek T, Picard J, Weinberg G. LIPAEMIC report: results of clinical use of intravenous lipid emulsion in drug toxicity reported to an online lipid registry. J Med Toxicol. 2014;10:133-42.

6. Fettiplace MR, Weinberg G. The mechanisms underlying lipid resuscitation therapy. Reg Anesth Pain Med. 2018;43:138-49.

7. Weinberg GL, VadeBoncouer T, Ramaraju GA, Garcia-Amaro MF, Cwik MJ. Pretreatment or resuscitation with a lipid infusion shifts the dose-response to bupivacaine-induced asystole in rats. Anesthesiology. 1998;88:1071-5.

8. Dureau P, Charbit B, Nicolas N, Benhamou D, Mazoit JX. Effect of intralipid ${ }^{\circledR}$ on the dose of ropivacaine or levobupivacaine tolerated by volunteers: a clinical and pharmacokinetic study. Anesthesiology. 2016;125:474-83.

9. Shi K, Xia Y, Wang Q, Wu Y, Dong X, Chen C, Tang W, Zhang Y, Luo M, Wang X, Papadimos TJ, Xu X. The effect of lipid emulsion on pharmacokinetics and tissue distribution of bupivacaine in rats. Anesth Analg. 2013;116:804-9.

10. Gosmanov AR, Smiley DD, Peng L, Siquiera J, Robalino G, Newton C, Umpierrez GE. Vascular effects of intravenous intralipid and dextrose infusions in obese subjects. Metabolism. 2012;61:1370-6.

11. Partownavid P, Umar S, Li J, Rahman S, Eghbali M. Fatty-acid oxidation and calcium homeostasis are involved in the rescue of bupivacaine-induced cardiotoxicity by lipid emulsion in rats. Crit Care Med. 2012;40:2431-7.

12. Kuo I, Akpa BS. Validity of the lipid sink as a mechanism for the reversal of local anesthetic systemic toxicity: a physiologically based pharmacokinetic model study. Anesthesiology. 2013;118:1350-61.

13. Fettiplace MR, Akpa BS, Ripper R, Zider B, Lang J, Rubinstein I, Weinberg G. Resuscitation with lipid emulsion: dose-dependent recovery from cardiac pharmacotoxicity requires a cardiotonic effect. Anesthesiology. 2014;120:915-25.

14. Fettiplace MR, Ripper R, Lis K, Lin B, Lang J, Zider B, Wang J, Rubinstein I, Weinberg G. Rapid cardiotonic effects of lipid emulsion infusion. Crit Care Med. 2013;41:e156-62.

15. Maurice JM, Gan Y, Ma FX, Chang YC, Hibner M, Huang Y. Bupivacaine causes cytotoxicity in mouse $\mathrm{C} 2 \mathrm{C} 12$ myoblast cells: involvement of ERK and Akt signaling pathways. Acta Pharmacol Sin. 2010;31:493-500.

16. Cittadini A, Monti MG, Iaccarino G, Di Rella F, Tsichlis PN, Di Gianni A, Stromer H, Sorriento D, Peschle C, Trimarco B, Sacca L, Condorelli G. Adenoviral gene transfer of Akt enhances myocardial contractility and intracellular calcium handling. Gene Ther. 2006;13:8-19.

17. Fettiplace MR, Kowal K, Ripper R, Young A, Lis K, Rubinstein I, Bonini M, Minshall R, Weinberg G. Insulin signaling in bupivacaine-induced cardiac toxicity: Sensitization during recovery and potentiation by lipid emulsion. Anesthesiology. 2016;124:428-42.

18. Tobe M, Suto T, Saito S. The history and progress of local anesthesia: multiple approaches to elongate the action. J Anesth. 2018;32:632-6.

19. Tsuchiya M, Mizutani K, Ueda W. Adding dextran to local anesthetic enhances analgesia. J Anesth. 2019;33:163.

20. Aggarwal N. Local anesthetics systemic toxicity association with exparel (bupivacaine liposome) - a pharmacovigilance evaluation. Expert Opin Drug Saf. 2018;17:581-7.

21. Schnabel A, Meyer-Friessem CH, Zahn PK, Pogatzki-Zahn EM. Ultrasound compared with nerve stimulation guidance for peripheral nerve catheter placement: a meta-analysis of randomized controlled trials. Br J Anaesth. 2013;111:564-72.

22. Sasaki H, Yamauchi M, Ninomiya T, Tatsumi H, Yamakage M. Possible utility of contrast-enhanced ultrasonography for detecting spread of local anesthetic in nerve block. J Anesth. 2017;31:365-73.

Publisher's Note Springer Nature remains neutral with regard to jurisdictional claims in published maps and institutional affiliations. 